\title{
A Generalization of Magic Squares with Applications to Digital Halftoning ${ }^{\star}$
}

\author{
Boris Aronov ${ }^{1 \star \star}$, Tetsuo Asano ${ }^{2 \star \star \star}$, Yosuke Kikuchi $^{3}$, Subhas C. Nandy ${ }^{4}$, \\ Shinji Sasahara ${ }^{5}$, and Takeaki Uno ${ }^{6}$ \\ 1 Polytechnic University, Brooklyn, NY 11201-3840, USA, http : / cis . poly . edu/ aronov \\ 2 JAIST, Tatsunokuchi, 923-1292 Japan, t-asano@jaist.ac.jp \\ 3 ERATO QCI Project, JST, Tokyo 113-0033, Japan, kikuchi@qci.jst.go.jp \\ 4 Indian Statistical Institute, Kolkata 700 108, India, nandysc@isical.ac.in \\ 5 Fuji Xerox Co., Ltd., Kanagawa 259-0157, Japan. shinji.sasahara@fujixerox.co.jp \\ 6 National Institute of Informatics (NII), Tokyo, 101-8430 Japan, uno@ni i.jp
}

\begin{abstract}
A semimagic square of order $n$ is an $n \times n$ matrix containing the integers $0, \ldots, n^{2}-1$ arranged in such a way that each row and column add up to the same value. We generalize this notion to that of a zero $k \times k$ discrepancy matrix by replacing the requirement that the sum of each row and each column be the same by that of requiring that the sum of the entries in each $k \times k$ square contiguous submatrix be the same. We show that such matrices exist if $k$ and $n$ are both even, and do not if $k$ and $n$ are are relatively prime. Further, the existence is also guaranteed whenever $n=k^{m}$, for some integers $k, m \geq 2$. We present a space-efficient algorithm for constructing such a matrix.

Another class that we call constant-gap matrices arises in this construction. We give a characterization of such matrices.

An application to digital halftoning is also mentioned.
\end{abstract}

Keywords: digital halftoning, discrepancy, latin square, magic square, matrix.

\section{Introduction}

A semimagic square is an $n \times n$ matrix filled with the numbers $0, \ldots, n^{2}-1$ in such a way that the sum of the numbers in each row and each column are the same. Magic squares and related classes of integer matrices have been studied extensively (for an exhaustive bibliography, see [7] and the references therein).

This paper generalizes the notion of a semimagic square by replacing the requirement that all row and column sums be the same by the analogous requirement for all $k \times k$ contiguous square submatrices; we call such $n \times n$ matrices zero $k \times k$-discrepancy matrices of order $(k, n)$. Let $\mathbb{N}(k, n)$ be the set of all such matrices. In this paper we prove that $\mathbb{N}(k, n)$ is non-empty if $k$ and $n$ are both even, and empty if they are relatively prime. Further, we show by an explicit construction that $\mathbb{N}\left(k, k^{m}\right) \neq \emptyset$ for any integers $k, m \geq 2$.

Another property plays an important role in the latter construction of zero $k \times k$-discrepancy matrices. A characterization of matrices with this property is also given in this paper.

Our investigation is motivated by an application described below, but intuitively we seek a matrix filled with distinct integers in an as uniform a manner as possible. The analogous geometric problem of distributing $n$ points uniformly in a unit square has been studied extensively in the literature $[6,9]$. Usually, a family of regions is introduced to evaluate the uniformity of a point distribution. If the points of an $n$ point set $P$ are uniformly distributed, for any region $R$ in the family the number of points in $R$ should be close to $\frac{1}{n}$ area $(R)$, where $\frac{1}{n}$ is the point density of $P$ in the entire square. Thus, the discrepancy of $P$ in $a$ region $R$ is defined as the difference between this value and the actual number of points of $P$ in $R$. The

* A prelininary version of this paper appeared in Proceedings of International Symposium on Algorithms and Computation, Hong Kong, December, 2004.

** Part of the work on the paper has been carried out when B.A. was visiting JAIST. Work of B.A. on this paper was supported in part by NSF ITR Grant CCR-00-81964.

$\star \star \star$ Work of T.A. was partially supported by the Ministry of Education, Science, Sports and Culture, Grant-in-Aid for Scientific Research (B). 
discrepancy of the point distribution $P$ with respect to the family of regions is defined by the maximum such difference, over all regions.

The problem of establishing discrepancy bounds for various classes of regions has been studied extensively [8]. One of the simplest families is that of axis-parallel rectangles for which $\Theta(\log n)$ bound is known [6,9]. In the context of digital halftoning, a family of axis-parallel squares (contiguous square submatrices) over a matrix is appropriate for measuring the uniformity since human eye perception is usually modeled using weighted sum of intensity levels with Gaussian coefficients over square regions around each pixel [2-4]. Thus, the matrices discussed in this paper can be used as dither matrices in which integers are arranged in an apparently random manner to be used as variable thresholds. Small matrix size tends to generate visible artifacts. In this sense the dither matrix of size $8 \times 8$ designed by Bayer [5] may be too small. A common way to construct a larger dither matrix is to use local search under some criterion based on spatial frequency distribution of the resulting matrix. Such dither matrices are called blue-noise masks [10-13]. One disadvantage of a blue-noise mask is its high space complexity. There appears to be no way to avoid storing the entire matrix. The zero $k \times k$-discrepancy matrices of order $\left(k, k^{n}\right)$ we construct, on the other hand, are such that we can generate any one element by a simple integer calculation requiring only $m$ seed matrices, each of size $k \times k$.

\section{Problem Statement}

Generalizing the notion of a semimagic square, we consider an $n \times n$ matrix containing all the integers $0, \ldots, n^{2}-1$ such that the entries contained in every contiguous $k \times k$ submatrix add up to the same value.

More formally, for integers $m, n>1$, let $\mathbb{Z}(n, m)$ be the class of all $n \times n$ integer matrices with entries from the set $\{0, \ldots, m-1\}$ and let $\mathbb{Z}(n) \subset \mathbb{Z}\left(n, n^{2}\right)$ be the set of those $n \times n$ matrices which contain every value $0, \ldots, n^{2}-1$ exactly once.

A contiguous $k \times k$ submatrix (or region, hereafter) $R_{i, j}=R_{i, j}^{(k)}$ with its upper left corner at $(i, j)$ is defined by

$$
R_{i, j}^{(k)}=\left\{\left(i^{\prime}, j^{\prime}\right) \mid i^{\prime}=i, \ldots, i+k-1 \text { and } j^{\prime}=j, \ldots, j+k-1\right\},
$$

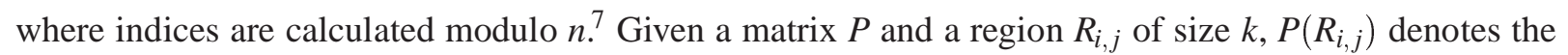
sum of the elements of $P$ in locations given by $R_{i, j}$. Analogously, define a $C_{i, j}=C_{i, j}^{(k)}$ to be the $k \times 1$ region of a matrix starting at $(i, j)$ and $P\left(C_{i, j}\right)$ to be the sum of elements of $P$ in the locations given by $C_{i, j}$. We are interested in all $k \times k$ regions in an $n \times n$ matrix:

$$
\mathscr{F}_{k, n}=\left\{R_{i, j}^{(k)} \mid i, j=0,1, \ldots, n-1\right\} .
$$

The $k \times k$-discrepancy $\mathscr{D}_{k, n}(P)$ of an $n \times n$ matrix $P$ for the family $\mathscr{F}_{k, n}$ is defined as

$$
\mathscr{D}_{k, n}(P)=\max _{R \in \mathscr{\mathscr { F }}_{k, n}} P(R)-\min _{R^{\prime} \in \mathscr{F}_{k, n}} P\left(R^{\prime}\right)
$$

In this paper we focus on the existence of matrices $P \in \mathbb{Z}(n)$ with $k \times k$-discrepancy $\mathscr{R}, n(P)=0$. In other words, we are interested in the existence and construction of matrices in $\mathbb{Z}(n)$ all of whose contiguous $k \times k$ submatrices have equal sums. Let $\mathbb{N}(k, n)$ be the set of all such zero- $k \times k$-discrepancy matrices of $\operatorname{order}(k, n)$.

Theorem 1. The set $\mathbb{N}(k, n)$ of zero- $k \times k$-discrepancy matrices of order $(k, n)$ has the following properties:

(a) $\mathbb{N}(k, n)$ is non-empty if $k$ and $n$ are both even.

(b) $\mathbb{N}(k, n)$ is empty if $k$ and $n$ are relatively prime.

(c) $\mathbb{N}(k, n)$ is empty if $k$ is odd and $n$ is even.

\footnotetext{
${ }^{7}$ Throughout this paper, index arithmetic is performed modulo matrix dimensions unless otherwise noted.
} 
(d) $\mathbb{N}\left(k, k^{m}\right)$ is non-empty for any integers $k$ and $m, k \geq 2, m \geq 2$.

In addition, using the above results, we can show that there is no $n \times n$ matrix $P$ that achieves zerodiscrepancy simultaneously for the families $\mathscr{F}_{2, n}$ and $\mathscr{F}_{3, n}$, i.e., $\mathbb{N}(2, n) \cap \mathbb{N}(3, n)=\emptyset$,

Proof (Theorem 1, parts (a)-(c)). To prove part (a), it suffices to show $\mathbb{N}(2, n) \neq \emptyset$ if $n$ is even since any $k \times k$ region can be partitioned into $2 \times 2$ regions if $k$ is even. (More generally, if $k$ divides $k, \mathbb{N}\left(k^{\prime}, n\right) \subset$ $\mathbb{N}(k, n)$.)

Let $P=\left(p_{i, j}\right) \in \mathbb{Z}(n)$ be the matrix in which the numbers are arranged in the row-major order, that is, $p_{i, j}=i n+j, i, j=0,1, \ldots, n-1$. We classify matrix elements by their parity and rotate all the elements

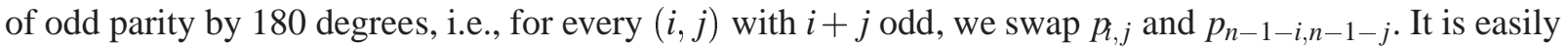
checked that the sum of elements in any $2 \times 2$ region is always $2 n^{2}-2$. An example for $n=8$ is shown in Fig. 1.

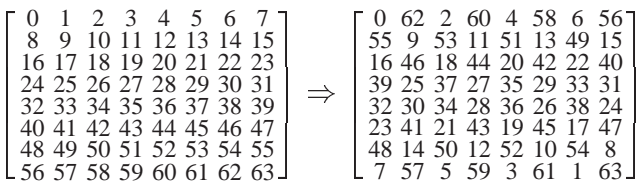

Fig. 1. Parity rotation used in the proof of Theorem 1(a).

Turning to part (b), for a contradiction, assume that there exists a matrix $P \in \mathbb{Z}(n)$ in which the sum $P\left(R_{i, j}\right)$ of elements of $P$ over a $k \times k$ region $R_{i, j}$ is independent of $i, j$. In particular, $P\left(R_{i, j}\right)=P\left(R_{i, j+1}\right)=c$ for some constant $c$ and therefore $P\left(C_{i, j}\right)=P\left(C_{i, j+k}\right)=c / k$, for all $i, j$.

Since $k$ and $n$ are relatively prime, the last relation implies that in fact $P\left(C_{i, j}\right)$ is independent of $j$. Similar reasoning leads to the conclusion that it is independent of $i$ as well. In particular, $P\left(C_{0,0}\right)=P\left(C_{1,0}\right)$, and therefore, by definition of $C_{0,0}$ and $C_{1,0}$, we must have $p_{i, 0}=p_{i+k, 0}$, contradicting our assumption that all the elements of $P$ are distinct.

Finally, we consider part (c) of Theorem 1. Let $P \in \mathbb{Z}(n)$ and let $k$ be odd and $n$ be even. For a contradiction, assume that the values in any $k \times k$ region add up to the same number, say $S$, which must clearly be an integer. Summing $P\left(R_{i, j}\right)$ over all $i$ and $j$ and observing that every entry in $P$ appears precisely $k^{2}$ times in these sums, we conclude that

$$
n^{2} S=k^{2}\left(0+1+\cdots+n^{2}-1\right)=k^{2} \frac{n^{2}}{2}\left(n^{2}-1\right),
$$

and therefore $S=k^{2}\left(n^{2}-1\right) / 2$, which cannot be an integer if $n$ is even and $k$ is odd. This contradiction concludes the proof of Theorem 1(a)-(c).

\section{Construction of a $k^{m} \times k^{m}$-Matrix of Zero $k \times k$-Discrepancy}

In this section we finish the proof of Theorem 1 by designing a $k^{m} \times k^{m}$ matrix from $\mathbb{Z}\left(k^{m}\right)$ for any positive integer $m$ such that its $k \times k$-discrepancy is zero; in fact we present a proof of a stronger statement, see Theorem 3. We first show that there exists a $k^{2} \times k^{2}$ matrix in $\mathbb{Z}\left(k^{2}\right)$ whose $k \times k$ discrepancy is zero, and then extend the result to $k^{m} \times k^{m}$ matrices.

Definition 1. The simple expansion $\tilde{P}$ of a $k \times k$ matrix $P$ is the matrix formed by repeating $P k \times k$ times, as follows:

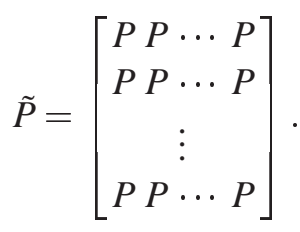


Note that the $k \times k$-discrepancy of $\tilde{P}$ is zero, as every $k \times k$ region contains the same set of numbers.

Definition 2. A cyclic column shift of a matrix $P$ is the matrix obtained by shifting each column of $P$ to the right (i.e., shifting the jth column to the $(j+1)$ st column) and moving the last column to the first column. A cyclic row shift is similarly defined: It means shifting each row of $P$ down to the next lower row (i.e., shifting ith row to the $(i+1)$ st row) and moving the bottom row to the top row.

We denote the matrix obtained by applying cyclic column shift $c$ times and cyclic row shift $r$ times to a $k \times k$ matrix $P$ by $P^{(c, r)}$. That is, element $(i, j)$ in $P$ moves to position $((i+r) \bmod k,(j+c) \bmod k)$ in $P^{(c, r)}$. The cyclic expansion $\hat{P}=\left(\hat{p}_{i, j}\right)$ of a $k \times k$ matrix $P$ is a $k^{2} \times k^{2}$ matrix defined by

$$
\hat{P}=\left[\begin{array}{llll}
P^{(0,0)} & P^{(0,1)} & \cdots & P^{(0, k-1)} \\
P^{(1,0)} & P^{(1,1)} & \cdots & P^{(1, k-1)} \\
& \vdots & & \\
& & \\
P^{(k-1,0)} & P^{(k-1,1)} & \cdots & P^{(k-1, k-1)}
\end{array}\right]
$$

An easy calculation shows that, for all $i, j, \hat{p}_{i, j}=p_{i^{\prime}, j^{\prime}}$, with

$$
i^{\prime}=i+\lfloor j / k\rfloor \text { and } j^{\prime}=j+\lfloor i / k\rfloor \quad(\bmod k) .
$$

Definition 3. A constant-gap matrix $P=\left(p_{i, j}\right)$ is one for which

$$
p_{i, j}-p_{i, j^{\prime}}=p_{i^{\prime}, j}-p_{i^{\prime}, j^{\prime}}
$$

holds for all choices of $i, i^{\prime}, j$, and $j^{\prime}$.

Intuitively, this means that for any two columns $j$ and $f$ the gap between elements in the same row is independent of the row, hence the "constant gap" name. Since (2) can be rewritten as

$$
p_{i, j}-p_{i^{\prime}, j}=p_{i, j^{\prime}}-p_{i^{\prime}, j^{\prime}} \text { or } p_{i, j}+p_{i^{\prime}, j^{\prime}}=p_{i, j^{\prime}}+p_{i^{\prime}, j}
$$

rows and columns play symmetric roles in the definition. Moreover, a constant-gap matrix has the strong Monge property [1] since the sum of the main diagonal elements is equal to that of the off diagonal elements in any $2 \times 2$ submatrix.

Lemma 1. The constant-gap property is preserved (1) under exchange of any two rows, (2) under exchange of any two columns, and, for square matrices, (3) under mirror reflection across the main diagonal.

Proof. Immediate from the definition.

The following lemma is a key to our construction of zero discrepancy matrices.

Lemma 2. If $P$ is a $k \times k$ constant-gap matrix, the $k \times k$-discrepancy of its cyclic expansion $\hat{P}$ is zero.

Proof. Recall that $R_{i, j}$ and $C_{i, j}$ denote $k \times k$ and $k \times 1$ contiguous submatrices of $\hat{P}$, and $\hat{P}\left(R_{i, j}\right)$ and $\hat{P}\left(C_{i, j}\right)$ the sums of the corresponding elements in $\hat{P}$, respectively. We aim to prove $\hat{P}\left(R_{i, j}\right)=\hat{P}\left(R_{i, j+1}\right)$, for all $i, j$. Together with $\hat{P}\left(R_{i, j}\right)=\hat{P}\left(R_{i+1, j}\right)$, which is proven by a symmetric argument, this implies the statement of the theorem. By definition, $\hat{P}\left(R_{i, j+1}\right)-\hat{P}\left(R_{i, j}\right)=\hat{P}\left(C_{i, j+k}\right)-\hat{P}\left(C_{i, j}\right)$; recall that all indices in $\hat{P}$ are calculated modulo $k^{2}$.

Put $i_{0}=k\lfloor i / k\rfloor$ and $j_{0}=k\lfloor j / k\rfloor$. To prove $\hat{P}\left(C_{i, j}\right)=\hat{P}\left(C_{i, j+k}\right)$ we compare the two columns. As illustrated in Fig. 2, the part above the element $\left(i_{0}+k-1, j\right)$ and the one above the element $(i+k-$ $1, j)$ in $C_{i, j}$ both appear in $C_{i, j+k}$. Differences between $C_{i, j}$ and $C_{i, j+k}$ comprise only four elements: $a=$ 


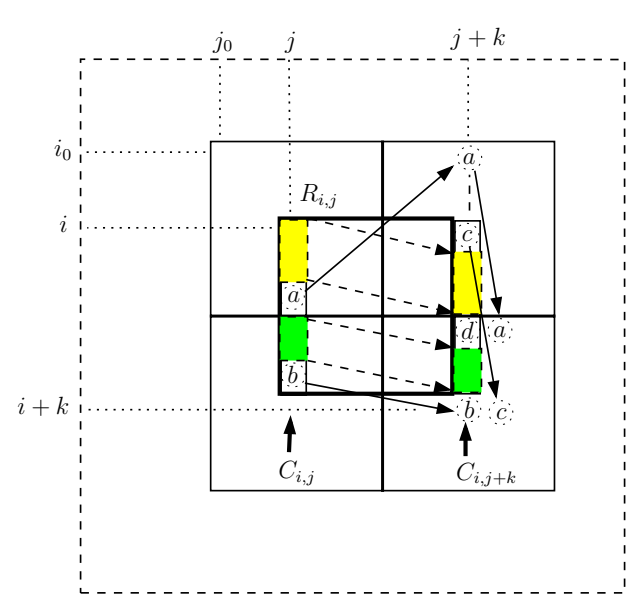

Fig. 2. Illustration to the proof of Lemma 2.

$\hat{p}_{i_{0}+k-1, j}, b=\hat{p}_{i+k-1, j}, c=\hat{p}_{i, j+k}, d=\hat{p}_{i_{0}+k, j+k}$. By cyclic row and column shifts, the four elements move in $\hat{P}$ as follows:

$$
\begin{aligned}
a & =\hat{p}_{i_{0}+k-1, j} \rightarrow_{r} \hat{p}_{i_{0}, j+k} \rightarrow_{c} \hat{p}_{i_{0}+k, j+k+1}, \\
b & =\hat{p}_{i+k-1, j} \rightarrow_{r} \hat{p}_{i+k, j+k}, \\
c & =\hat{p}_{i, j+k} \rightarrow_{c} \hat{p}_{i+k, j+k+1}, \\
d & =\hat{p}_{i_{0}+k, j+k},
\end{aligned}
$$

where $\rightarrow_{x}$ represents the cyclic $x$ shift and indices are calculated modulo $k^{2}$.

When $j \neq k-1(\bmod k)$ and $i \neq k-1(\bmod k)$, all four elements

$$
d: \hat{p}_{i_{0}+k, j+k}, a: \hat{p}_{i_{0}+k, j+k+1}, b: \hat{p}_{i+k, j+k}, \text { and } c: \hat{p}_{i+k, j+k+1}
$$

belong to the same submatrix, namely, to $P^{\left(\left\lfloor i_{0} / k\right\rfloor+1,\left\lfloor j_{0} / k\right\rfloor+1\right)}$. Since the constant gap property is preserved by cyclic row and column shifts, we have $d-b=a-c$, and thus $a+b=c+d$. It may happen that $j+k$ and $j+k+1$ belong to different contiguous submatrices. In fact, it happens when $j=k-1(\bmod k)$ and $i=k-1(\bmod k)$. If $j=k-1(\bmod k)$, we extend the sequence as follows:

$$
\begin{aligned}
a & =\hat{p}_{i_{0}+k-1, j} \rightarrow_{r} \hat{p}_{i_{0}, j+k} \rightarrow_{c} \hat{p}_{i_{0}+k, j_{0}+k} \rightarrow_{r} \hat{p}_{i_{0}+k+1, j_{0}+2 k}=\hat{p}_{i_{0}+k+1, j+k+1}, \\
b & =\hat{p}_{i+k-1, j} \rightarrow_{r} \hat{p}_{i+k, j+k}, \rightarrow_{r} \hat{p}_{i+k+1, j+2 k}, \\
c & =\hat{p}_{i, j+k} \rightarrow_{r} \hat{p}_{i+1, j+2 k}, \rightarrow_{c} \hat{p}_{i+k+1, j+k+1}, \\
d & =\hat{p}_{i_{0}+k, j+k} \rightarrow_{r} \hat{p}_{i_{0}+k+1, j+2 k} .
\end{aligned}
$$

Then, the four elements lie in the same contiguous submatrix as we required. The case for $i=k-1$ $(\bmod k)$ is similar. This completes the proof of $\hat{P}\left(C_{i, j}\right)=\hat{P}\left(C_{i, j+k}\right)$ and of the lemma.

Lemma 3. Let $P=\left(p_{i j}\right)$ and $Q=\left(q_{i j}\right)$ be matrices in $\mathbb{Z}(k)$. Combine $\hat{P}$ and $\tilde{Q}$ into a single matrix in two different ways, namely, put $C^{(1)}=C^{(1)}(P, Q)=\left(c_{i j}\right)=\tilde{Q}+k^{2} \hat{P}$ and $C^{(2)}=C^{(2)}(P, Q)=\left(c_{i j}^{\prime}\right)=\hat{P}+k^{2} \tilde{Q}$. In other words, $c_{i, j}=\tilde{q}_{i, j}+k^{2} \hat{p}_{i, j}$ or $c_{i, j}^{\prime}=\hat{p}_{i, j}+k^{2} \tilde{q}_{i, j}$, for all $i, j$. If $P$ has the constant gap property, then

(a) $C^{(1)}$ and $C^{(2)}$ are in $\mathbb{Z}\left(k^{2}\right)$, and

(b) their $k \times k$-discrepancy is zero.

In addition, $C^{(1)}$ and $C^{(2)}$ are distinct if $P \neq Q$. Thus $\left|\mathbb{N}\left(k, k^{2}\right)\right| \geq 2$. 
Proof. The resulting matrices obviously belong to $\mathbb{Z}\left(k^{2}, k^{4}\right)$ and have zero discrepancy, as linear combinations of matrices of zero discrepancy. It is easy to check that $C^{(1)} \neq C^{(2)}$ if $P \neq Q$.

Thus to prove (b), it suffices to show that the elements of the matrices are all distinct. We focus on $C^{(1)}$, the argument for $C^{(2)}$ is analogous. Since $\hat{P}, \tilde{Q} \in \mathbb{Z}\left(k^{2}, k^{2}\right), c_{i j}=c_{i^{\prime} j^{\prime}}$ implies $\hat{p}_{i j}=\hat{p}_{i^{\prime} j^{\prime}}$ and $\tilde{q}_{i j}=\tilde{q}_{i^{\prime} j^{\prime}}$. In other words, for a repeated value to occur in $C^{(1)}$, there must exist two positions $(i, j)$ and $\left(t, j^{\prime}\right)$ so that in $\hat{P}$ the same number occurs at $(i, j)$ and $\left(l^{\prime}, j^{\prime}\right)$, and this also happens in $\tilde{Q}$. We argues that this is impossible. Indeed, since $\tilde{Q}$ is defined by just repeating the same matrix (with all entries distinct) $k^{2}$ times, each element stays in the same relative position in each submatrix. On the other hand, no element in a submatrix $P^{(c, r)}$ of $\hat{P}$ occurs in the same position in any other submatrix.

We now prove a stronger version of Theorem $1 \mathrm{~d}$.

Theorem 2. $\mathbb{N}\left(k, k^{m}\right) \neq$ for any integers $k, m \geq 2$. Moreover, a zero-discrepancy matrix in $\mathbb{N}\left(k, k^{m}\right)$ can be explicitly computed in time linear in its size using $O\left(m k^{2}\right)$ space.

Proof. We generalize the construction presented in Lemma 3. A matrix $M \in \mathbb{Z}\left(k^{m}\right)$ with zero discrepancy is defined using $m-1$ constant-gap matrices $P_{0}, P_{1}, \ldots, P_{m-2}$ of size $k$ and one arbitrary matrix $P_{m-1}$ of the same size (all in $\mathbb{Z}(k)$ ) as follows:

$$
\begin{aligned}
M(i, j)= & k^{2(m-1)} P_{0}^{\left(\left\lfloor i / k^{m-1}\right\rfloor,\left\lfloor j / k^{m-1}\right\rfloor\right)}(i \bmod k, j \bmod k) \\
& +k^{2(m-2)} P_{1}^{\left(\left\lfloor i / k^{m-2}\right\rfloor \bmod k,\left\lfloor j / k^{m-2}\right\rfloor \bmod k\right)}(i \bmod k, j \bmod k) \\
& \cdots \\
& +k^{2} P_{m-2}^{(\lfloor i / k\rfloor \bmod k,\lfloor j / k\rfloor \bmod k)}(i \bmod k, j \bmod k) \\
& +P_{m-1}(i \bmod k, j \bmod k)
\end{aligned}
$$

For example, when $k=3$ and $m=3, M$ is constructed as follows, where we have used $R, Q, P$ for $P_{0}, P_{1}, P_{2}$, respectively, to avoid cumbersome notation.

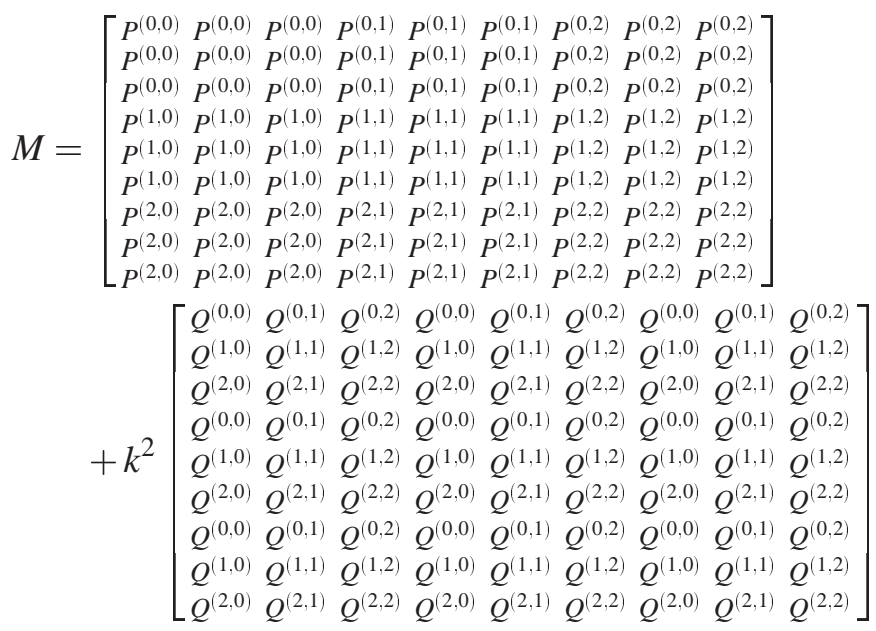

$$
\begin{aligned}
& +k^{4}\left[\begin{array}{lllllllll}
R & R & R & R & R & R & R & R & R \\
R & R & R & R & R & R & R & R & R \\
R & R & R & R & R & R & R & R \\
R & R & R & R & R & R & R & R & R \\
R & R & R & R & R & R & R & R & R \\
R & R & R & R & R & R & R & R & R \\
R & R & R & R & R & R & R & R \\
R & R & R & R & R & R & R & R & R \\
R & R & R & R & R & R & R & R & R
\end{array}\right] .
\end{aligned}
$$

The remainder of the proof proceeds just as in that of Lemma 3; we omit the details. Recall that $P_{m}^{(a, b)}(i, j)=P_{m}((i+b) \bmod k,(j+a) \bmod k)$. Thus we can generate every entry of such a matrix without explicitly storing any information besides the $m k \times k$ matrices $P, \ldots, P_{m-1}$; the computation requires at most $O(m)$ additional working space. 


$\left[\begin{array}{lll}1 & 2 & 3 \\ 4 & 5 & 6 \\ 7 & 8 & 9\end{array}\right] \quad\left[\begin{array}{cccc}0 & 1 & 2 & 3 \\ 4 & 5 & 6 & 7 \\ 8 & 9 & 10 & 11 \\ 12 & 13 & 14 & 15\end{array}\right] \quad\left[\begin{array}{cccc}0 & 1 & 4 & 5 \\ 2 & 3 & 6 & 7 \\ 8 & 9 & 12 & 13 \\ 10 & 11 & 14 & 15\end{array}\right] \quad\left[\begin{array}{cccc}0 & 1 & 8 & 9 \\ 2 & 3 & 10 & 11 \\ 4 & 5 & 12 & 13 \\ 6 & 7 & 14 & 15\end{array}\right]$

Fig. 3. The only equivalence class of constant-gap matrices for $n=3$ (a), and the three classes for $n=4$ (b)

\section{The Class of Constant-Gap Matrices}

We have described a scheme for constructing matrices with zero $k \times k$-discrepancy. A key ingredient in the recipe is a constant-gap matrix in $\mathbb{Z}(k)$. It is easily checked that a different choice of such a matrix produces a different zero-discrepancy matrix. Thus a natural question arises: How many different constantgap matrices of a given size are there? In this section we, in a sense, completely characterize the class of constant-gap matrices in $\mathbb{Z}(n)$. In fact, we discuss a somewhat more general class of matrices. Let $\mathbb{M}(m, n)$ be the set of all integer $m \times n$ matrices with entries $0, \ldots, m n-1$, each used exactly once. A matrix $M=\left(m_{i j}\right) \in \mathbb{M}(m, n)$ has constant-gap property if, for all $i, j, l^{\prime}, j^{\prime}, m_{i j}+m_{i^{\prime} j^{\prime}}=m_{i^{\prime} j}+m_{i j^{\prime}}$. It is clear from the definition that this property, as already observed in Lemma 1, is invariant under a number of operations:

Lemma 4. The constant-gap property is preserved (1) under an arbitrary permutation of rows of a matrix, (2) under an arbitrary permutation of columns of a matrix, and, for square matrices, (3) under mirror reflection through its main diagonal.

Two constant-gap matrices are equivalent if one of them is derived from the other by a sequence of operations listed in the statement of the lemma. ${ }^{8}$ We are interested in counting the number of these equivalence classes.

Lemma 5. Every equivalence class can be represented by a matrix $P=\left(p_{i, j}\right) \in \mathbb{M}(m, n)$ in canonical form, which satisfies the following additional properties:

(a) Every row of $P$ is sorted, i.e., $p_{i, 0}<p_{i, 1}<\cdots<p_{i, n-1}$; for convenience we define $c_{j}=p_{0, j}$ for $j=0, \ldots, n-1$.

(b) Every column of $P$ is sorted, i.e., $p_{0, j}<p_{1, j}<\cdots<p_{n-1, j}$; we put $r_{j}=p_{j, 0}$, for $j=0, \ldots, n-1$.

(c) Generally, $p_{i, j}<p_{i^{\prime}, j^{\prime}}$ if $i \leq i^{\prime}, j \leq j^{\prime}$, and $(i, j) \neq\left(i^{\prime}, j^{\prime}\right)$.

(d) $p_{0,0}=c_{0}=r_{0}=0, p_{m, n}=m n-1$.

(e) $P$ is completely specified by $\left(c_{j}\right)$, $\left(r_{i}\right):$ for all $i, j, p_{i, j}=r_{i}+c_{j}$.

Proof. We argue that all the properties can be satisfied without leaving the equivalence class. Columns can be permuted to sort the top row and then rows can be permuted to sort the leftmost column. Because of the constant-gap property, this sorts all rows and columns. Properties (c) and (d) follow from this ordering. The next property follows from the constant-gap condition, namely $p_{, j}=p_{0,0}+p_{i, j}=p_{i, 0}+p_{0, j}=r_{i}+c_{j}$. The final property can be ensured for square matrices by taking, if necessary, a reflection through the main diagonal.

It is not difficult to see that there exists only one equivalence class (up to diagonal reflection) of constant-gap matrices in $\mathbb{Z}(n)$ when $n=3$; for $n=4$, there are three equivalence classes, refer to Fig. 3. For $n=5$ there exists only one equivalence class, whereas there are many when $n=6$. These observations can be generalized as follows.

\footnotetext{
${ }^{8}$ To avoid cumbersome wording, we will henceforth not discuss the case of square matrices separately. The reasoning there is entirely analogous, with the exception of an occasional invocation of diagonal symmetry to further reduce the number of equivalence classes. We omit further details.
} 
The set of all constant-gap matrices in $\mathbb{M}(m, n)$ in canonical form is denoted by $\mathbb{K}(m, n)$. In this section we give a characterization of the sets $\mathbb{K}(m, n)$, for all $m, n>0$. We begin with some additional terminology and then state our characterization.

We define another operation on matrices. Given matrices $P=\left(p_{i, j}\right) \in \mathbb{K}(m, n)$ and $Q=\left(q_{i, j}\right) \in$ $\mathbb{K}\left(m^{\prime}, n^{\prime}\right)$, their expansion product $P \otimes Q$ is the $m m^{\prime} \times n n^{\prime}$ matrix $H=\left(h_{i, j}\right)$ defined by

$$
h_{i, j}=m^{\prime} n^{\prime} p_{\left\lfloor i / m^{\prime}\right\rfloor,\left\lfloor j / n^{\prime}\right\rfloor}+q_{i \bmod m^{\prime}, j \bmod n^{\prime}}
$$

In addition, define a simple row of length $k$ to be the $1 \times k$ matrix filled with consecutive numbers $0, \ldots, k-$ 1 , in this order. Define a simple column analogously.

The following facts are easily verified.

Fact 1. $\mathbb{K}(1, m)$ consists of a single matrix, which is a simple row of length $m$. An analogous statement holds for $\mathbb{K}(m, 1)$. Both row- and column-major order filled members of $\mathbb{M}(m, n)$ have constant-gap property. In particular, $|\mathbb{K}(m, n)| \geq 2$, for $m, n>1$. The two matrices have the same canonical form in $\mathbb{K}(n)$, so $|\mathbb{K}(n)| \geq 1$ for $n \geq 1$.

Fact 2. If $P \in \mathbb{K}(m, n)$ and $Q \in \mathbb{K}\left(m^{\prime}, n^{\prime}\right)$, then $P \otimes Q \in \mathbb{K}\left(m m^{\prime}, n n^{\prime}\right)$.

Surprisingly, in the sense made more precise by the following theorem, Facts 1 and 2 describe $\mathbb{K}(m, n)$ completely. The remainder of the section is devoted to the proof of this assertion.

Theorem 3. $\mathbb{K}(m, n)$ can be characterized as follows.

(a) A matrix $P \in \mathbb{K}(m, n)$ can be written uniquely as

$$
P=P_{1} \otimes \cdots \otimes P_{k},
$$

where $P_{1}, \ldots, P_{k}$ is an alternating sequence of simple rows and columns, each of length at least two; $k=0$ if $m=n=1$.

(b) $\mathbb{K}(m, n) \neq \emptyset$, for $m, n>0$. $|\mathbb{K}(m, n)|=1$ if and only if $n=1$ or $m=1$. In this case, $\mathbb{K}(m, n)$ consists just a simple row or column.

A column difference $p_{0, j}-p_{0, k}=p_{i, j}-p_{i, k}$, for some $j \neq k$, is the difference between corresponding entries of two columns; it is independent of the row where the difference is taken, by the constant-gap property. Similarly, a row difference $p_{i, 0}-p_{k, 0}=p_{i, j}-p_{k, j}$, for $i \neq k$, is the difference between corresponding entries of two rows.

Lemma 6. No row difference equals a column difference. In other words, for any $0 \leq i<k \leq n-1$ and $0 \leq j<\ell \leq n-1, p_{0, k}-p_{0, i} \neq p_{\ell, 0}-p_{j, 0}$.

Proof. By the constant-gap property, $p_{0, k}-p_{0, i}=p_{\ell, k}-p_{\ell, i}$ and $p_{\ell, 0}-p_{j, 0}=p_{\ell, k}-p_{j, k}$. Their equality would imply $p_{\ell, i}=p_{j, k}$ contradicting the assumption that no entry in $P$ is repeated.

Proof (Theorem 3). We start with the existence proof for part (a). If $n=1$ or $m=1$, we are done, so assume $m, n>1$. Without loss of generality, assume that $p_{0,1}<p_{1,0}$, so that $p_{0,1}=1$. By induction, it is sufficient to argue that in this case $P$ can be written as a product of a smaller matrix and a simple row of length at least two.

Let $P=\left(p_{i, j}\right) \in \mathbb{K}(m, n)$. A horizontal run in $P$ (an $h$-run, for short) is a maximal sequence of consecutive integers appearing in adjacent entries of a row of $P$; the length of an h-run is the number of such integers. Each h-run is associated with an interval defined by its first and last column indices. The initial $h$-run is the one starting at the upper left corner $p_{0,0}$ of the matrix; by our assumption its length $\ell$ is at least two. The value $\ell$ must be contained in $p_{0,1}$, for it cannot lie in $p_{0, \ell}$ by maximality of the run and it is the smallest value in the matrix outside of the run. Thus we have the row difference $p_{1,0}-p_{0,0}=\ell$. 


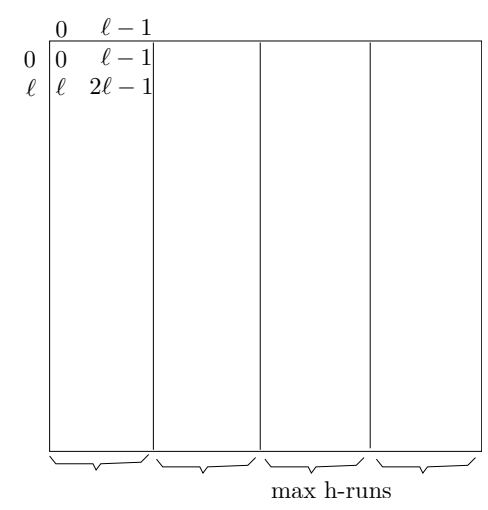

Fig. 4. Partition of a matrix by vertical boundaries of h-runs.

\section{Lemma 7. No h-run is longer than the initial run.}

Proof. If there existed such an h-run, we would have a column difference equal to $\ell$ appear within the run. However, we already identified a row difference of $\ell$ in the matrix. This would contradict Lemma 6.

Recall that in $P$ the differences between consecutive elements in the row are equal to the corresponding differences in the top row, so in terms of presence and length of runs, every row behaves exactly the same. Thus $P$ can be partitioned by vertical lines corresponding to boundaries of h-runs, as shown in Fig. 4 .

Lemma 8. All h-runs have the same length.

Proof. By Lemma 7, no h-run is longer than the initial h-run of length $\ell$. For a contradiction, let $j$ be the first column at which an h-run of length $\ell<\ell$ starts. Such an h-run in the top row is a sequence $p_{0, j}, p_{0, j}+1, \ldots, p_{0, j}+\ell^{\prime}-1$. Where in the matrix is the next integer, namely the value $q=p_{0, j}+\ell^{\prime}$ ? By definition of an h-run, it cannot be located in the same row. So, it must lie before column $j$, say in location $p_{k, s \ell}$, for some $s \ell<j$. Since all the h-runs located to the left of column $j$ are of length $\ell$, the h-run starting at $p_{k, s \ell}$ must consist of values $q, \ldots, q+\ell-1$. However, since the difference between rows zero and one is $\ell, p_{1, j}$ must have value $r=p_{0, j}+\ell>q$. Moreover, $r=q-\ell^{\prime}+\ell<q+\ell-1$, so the value $r$ occurs both at $p_{1, j}$ and in the run starting at value $q$, which is impossible.

This property implies the following structure of the matrix $P$. The number $\ell>1$ is a divisor of $n$. The entire matrix consists of h-runs of length $\ell$. The first element of each run is a multiple of $\ell$. If $L$ is a simple row of length $\ell$, we can easily verify that indeed $P=P \otimes \ell$ for the matrix $P^{\prime}=\left(p_{i, j}^{\prime}\right)$ defined by $p_{i, j}^{\prime}=p_{i, \ell j} / \ell$. We claim that $P^{\prime} \in \mathbb{K}(m, n / \ell)$. It is easily checked that $P^{\prime} \in \mathbb{M}(m, n / \ell)$, while properties (a)(e) follow from the corresponding properties for $P$.

We now address the question of uniqueness. We have shown that any matrix in $\mathbb{K}(m, n)$ can we written as an expansion product of some number of simple rows and columns. Since the expansion product of two simple rows (resp. columns) is a simple row (resp. column), by consolidating products of consecutive rows (resp. columns) we can express $P$ as a product of alternating rows and columns. This representation is unique, since the type and size of the last factor can be "read" directly from the matrix - the factor is a simple row if $p_{0,1}=1$ and a simple column if $p_{1,0}=1$; its length $\ell$ is the length of the initial run, which is horizontal in the former case and vertical in the latter one.

It remains to note that a matrix from $\mathbb{K}(m, n)$ can always be produced as a product of a simple row of length $n$ and a simple column of length $m$, thus constant-gap matrices of all orders exist. Reversing the order of multiplication produces a different matrix, provided $m, n>1$, proving part (b) of the theorem.

\section{Concluding Remarks}

We have introduced a discrepancy-based measure of uniformity of an $n \times n$ square matrix containing $0,1, \ldots, n^{2}-1$ as a generalization of a semimagic square. We have succeeded in obtaining matrices of even 
dimension with zero discrepancy for families of $2 k \times 2 k$ contiguous submatrices. For arbitrary $k$, we can construct a $k^{m} \times k^{m}$ matrix of $k \times k$-discrepancy zero. Moreover, such a matrix can be explicitly computed in time linear in its size using only $O\left(m k^{2}\right)$ space, which is a great advantage over the heuristic algorithms used for designing blue-noise masks in digital halftoning. This paper serves as a starting point of this type of investigation. A number of issues are still left open. One of the most interesting and attractive problems is to find low-discrepancy matrices, when $n$ (dimension of the matrix) and $k$ (the dimension of submatrix) are relatively prime to each other.

\section{References}

1. A. Aggarwal, M. Klawe, S. Moran, P. Shor, and R. Wilber: "Geometric Applications of a Matrix Searching Algorithm," Proc. 2nd ACM Symposium on Computational Geometry, pp. 285-292, 1986.

2. T. Asano, "Digital Halftoning: Algorithm Engineering Challenges," IEICE Trans. on Inf. and Syst., E86-D, 2, 159-178, 2003.

3. T. Asano, K. Obokata, N. Katoh, and T. Tokuyama: "Matrix rounding under the $L_{p}$-discrepancy measure and its application to digital halftoning," Proc. ACM-SIAM Symposium on Discrete Algorithms, pp. 896-904, San Francisco, 2002.

4. T. Asano, N. Katoh, K. Obokata, and T. Tokuyama, "Combinatorial and Geometric Problems Related to Digital Halftoning," Theoretical Foundations of Computer Vision: Geometry, Morphology, and Computational Imaging, LNCS 2616, Springer, 2003.

5. B.E. Bayer: "An optimum method for two-level rendition of continuous-tone pictures," Conference Record, IEEE International Conference on Communications, 1, pp. (26-11)-(26-15), 1973.

6. B. Chazelle: The Discrepancy Method: Randomness and Complexity, Cambridge University Press, 2000.

7. H.D. Heinz: "Magic Squares, Magic Stars \& Other Patterns" web site, http://www.geocities.com/CapeCanaveral/Launchpad/ 4057/.

8. D.E. Knuth, The Art of Computer Programming, volume 1, Fundamental Algorithms, 3rd ed. (Reading, Massachusetts: Addison-Wesley, 1997.

9. J. Matoušek: Geometric Discrepancy, Springer, 1991.

10. T. Mitsa and K.J. Parker: "Digital halftoning technique using a blue-noise mask," J. Opt. Soc. Am., A/Vol. 9, No. 11, 19201929, 1992.

11. R.A. Ulichney: "Dithering with blue noise," Proc. IEEE, 76, 1, 56-79, 1988.

12. R. Ulichney: "The void-and-cluster method for dither array generation," IS\&T/SPIE Symposium on Electronic Imaging Science and Technology, Proceedings of Conf. Human Vision, Visual Processing and Digital Display IV, (Eds. Allebach, John Wiley), SPIE vol.1913, pp. 332-343, 1993.

13. M. Yao and K.J. Parker: "Modified approach to the construction of a blue noise mask," J. Electronic Imaging, 3, 92-97, 1994. 